\title{
Mitochondrial disease, mitophagy, and cellular distress in methylmalonic acidemia
}

\author{
Alessandro Luciani ${ }^{1} \cdot$ Matthew C. S. Denley ${ }^{2} \cdot$ Larissa P. Govers $^{1} \cdot$ Vincenzo Sorrentino $^{3} \cdot$ D. Sean Froese ${ }^{2}$
}

Received: 26 April 2021 / Revised: 18 August 2021 / Accepted: 30 August 2021 / Published online: 15 September 2021

(c) The Author(s) 2021

\begin{abstract}
Mitochondria - the intracellular powerhouse in which nutrients are converted into energy in the form of ATP or heat-are highly dynamic, double-membraned organelles that harness a plethora of cellular functions that sustain energy metabolism and homeostasis. Exciting new discoveries now indicate that the maintenance of this ever changing and functionally pleiotropic organelle is particularly relevant in terminally differentiated cells that are highly dependent on aerobic metabolism. Given the central role in maintaining metabolic and physiological homeostasis, dysregulation of the mitochondrial network might therefore confer a potentially devastating vulnerability to high-energy requiring cell types, contributing to a broad variety of hereditary and acquired diseases. In this Review, we highlight the biological functions of mitochondrialocalized enzymes from the perspective of understanding — and potentially reversing — the pathophysiology of inherited disorders affecting the homeostasis of the mitochondrial network and cellular metabolism. Using methylmalonic acidemia as a paradigm of complex mitochondrial dysfunction, we discuss how mitochondrial directed-signaling circuitries govern the homeostasis and physiology of specialized cell types and how these may be disturbed in disease. This Review also provides a critical analysis of affected tissues, potential molecular mechanisms, and novel cellular and animal models of methylmalonic acidemia which are being used to develop new therapeutic options for this disease. These insights might ultimately lead to new therapeutics, not only for methylmalonic acidemia, but also for other currently intractable mitochondrial diseases, potentially transforming our ability to regulate homeostasis and health.
\end{abstract}

Keywords Cell damage $\cdot$ Inherited metabolic diseases $\cdot$ Metabolism $\cdot$ Mitochondria $\cdot$ Mitophagy $\cdot$ Oxidative stress

\section{Introduction}

Alessandro Luciani and D. Sean Froese are lead contacts.

Alessandro Luciani

alessandro.luciani@uzh.ch

$\triangle$ Vincenzo Sorrentino

vincenzo.sorrentino@rd.nestle.com

$\triangle$ D. Sean Froese

sean.froese@kispi.uzh.ch

1 Mechanisms of Inherited Kidney Diseases Group, Institute of Physiology, University of Zurich, 8032 Zurich, Switzerland

2 Division of Metabolism and Children's Research Center, University Children's Hospital Zurich, University of Zurich, 8032 Zurich, Switzerland

3 Department of Musculo-Skeletal Health, Nestlé Institute of Health Sciences, Nestlé Research, 1015 Lausanne, Switzerland
Mitochondria are complex dynamic organelles that take the pilot's seat in the biology of most eukaryotic cells by playing a key part in catabolic and anabolic metabolism, hence guiding cell-wide programs for cellular growth and organismal homeostasis. Ever since its initial description by Altmann [1] and Benda [2] in the 1890s, the mitochondrion has come to be known as a double-membraneenclosed cytoplasmic organelle responsible for the generation of cellular energy through oxidative phosphorylation (OXPHOS) [3]. In parallel with its role in the production of cellular energy in the form of ATP, this highly dynamic organelle regulates intracellular calcium [4-6] and redox homeostasis [7, 8]. It also acts as a cardinal platform to coordinate molecular circuitries, toggling the balance between cell survival and regulated cell death pathways in response to changes in the surrounding microenvironment or stress conditions, hence driving nearly every aspect of 
cell differentiation, fate, and function [9]. Given the central role in preserving cellular and physiological homeostasis, imbalances in mitochondrial function thus pose a potentially devastating threat to many different cell types, fuelling pathologies associated with ageing, neurodegeneration, metabolic disease, and cancer [10-13].

Over the last two decades, studies of rare inherited genetic diseases, in combination with advances in technology and foundational genomic resources enabled by the high-throughput omics era, have provided novel insights into fundamental principles governing the contribution of mitochondria to cellular homeostasis and physiology. Through converging approaches, these pathway paradigms have captured a holistic understanding of the pathogenesis of other common human disorders in which mitochondrial dysfunction has been implicated as a secondary event in the progression of the disease. Furthermore, genome-wide association studies of urinary metabolites have identified the association between common sequence variants within or close to the genes encoding enzymes and transport proteins that enrich the mitochondrial matrix - the subcellular compartment in which many detoxification reactions and fatty acid and amino acid metabolism occur-and kidneyrelated traits (i.e. urine albumin-to-creatinine ratio and estimated glomerular filtration rate [13]) or different disease states, including chronic kidney disease [14]. These large-scale and multi-omics approaches thus support the fundamental role of mitochondria in organizing cellular homeostasis and organismal physiology, and its contribution to disease risk within the population.

In this review, we discuss the role of the mitochondria, not only as an energetic powerhouse, but also as a hub for signaling and homeostasis pathways and delve into the breakdown and removal of dysfunctional mitochondria through degradative processes. We further investigate diseases specifically affecting the mitochondria and take the dysfunction of the mitochondrial matrix residing metabolic enzyme methylmalonyl-coenzyme A mutase (MMUT) as a paradigm of mitochondrial disease. We describe the multiple roles of this enzyme in regulating the mitochondrial network and possible molecular and cellular pathways through which the loss-of-function mutations in MMUT might lead to mitochondrial distress, resulting in organ dysfunction primarily affecting the brain and kidney, and ultimately causing life -threatening manifestations. This Review also places a special emphasis on emerging advances in (patient-derived) cell-based and novel preclinical animal-based models that are empowering the development of small molecule-based drug discovery and screening programs. In the concluding section, we highlight regulatory pathways targeting cellular adversities linked to MMUT dysfunction and potential targets for therapeutically treating this life-threatening disease.

\section{Mitochondria: from structure to physiology to organelle quality control}

\section{Structure}

Mitochondria, whose name was coined by Carl Benda [2] in 1898 by combining the Greek words "mitos" (meaning thread) and "chondrion" (meaning granule), can be either found as isolated organelles or form large networks. They can have different morphological shapes and ultrastructural organization and distribute unevenly in the cytoplasm through specialized transport and positioning to adequately respond to changes in microenvironmental cues [15].

These highly dynamic and plastic organelles harbor multiple copies of their own bacterium-derived mitochondrial DNA (mtDNA) in the form of circular doublestranded DNA molecules. The mammalian mitochondriaderived proteome is composed of approximately 1500 proteins $[16,17]$ nearly all of which are encoded by nuclear DNA, translated in the cytosol, and delivered to mitochondrial sub-compartments through regulated import systems, processing, and assembly. By contrast, mtDNA only codifies approximately $1 \%$ of the mitochondrial proteome; these latter proteins being components of OXPHOS complexes [18]. Moreover, mtDNA mutations, whose rate is higher than the nuclear genome, can lead to metabolic dyshomeostasis, with defects in cellular energy production and tissue dysfunction, as testified by the identification of a large number of mitochondrial diseases [19]. Concomitantly, mutations in mtDNA might also accumulate during aging, wreaking havoc, directly or indirectly, on mitochondrial health and functionality, eventually contributing to age-induced adversities of a multitude of pathologies [18].

Mitochondria are composed of two structurally and functionally different membranes (Fig. 1), described as the outer mitochondrial membrane (OMM) and the inner mitochondrial membrane (IMM). The smooth and permeable OMM acts as a platform for the transmission and decoding of signaling cascades into the mitochondria. Beyond its primordial function as a barrier and its latter evolution into a signaling hub, the OMM also establishes a networks of physical and functional interactions with other intracellular moieties (Fig. 1) - such as the endoplasmic reticulum (ER), endosome and lysosome, peroxisome, plasma membrane, and lipid droplets-through the formation of membrane contact sites where the exchange of ions, metabolites, and other molecules can take place. Further details on the composition, dynamics, identity, and specific functions of membrane contact sites have been exhaustively reviewed elsewhere [20, 21]. By contrast, the IMM, which surrounds the mitochondrial matrix, is 


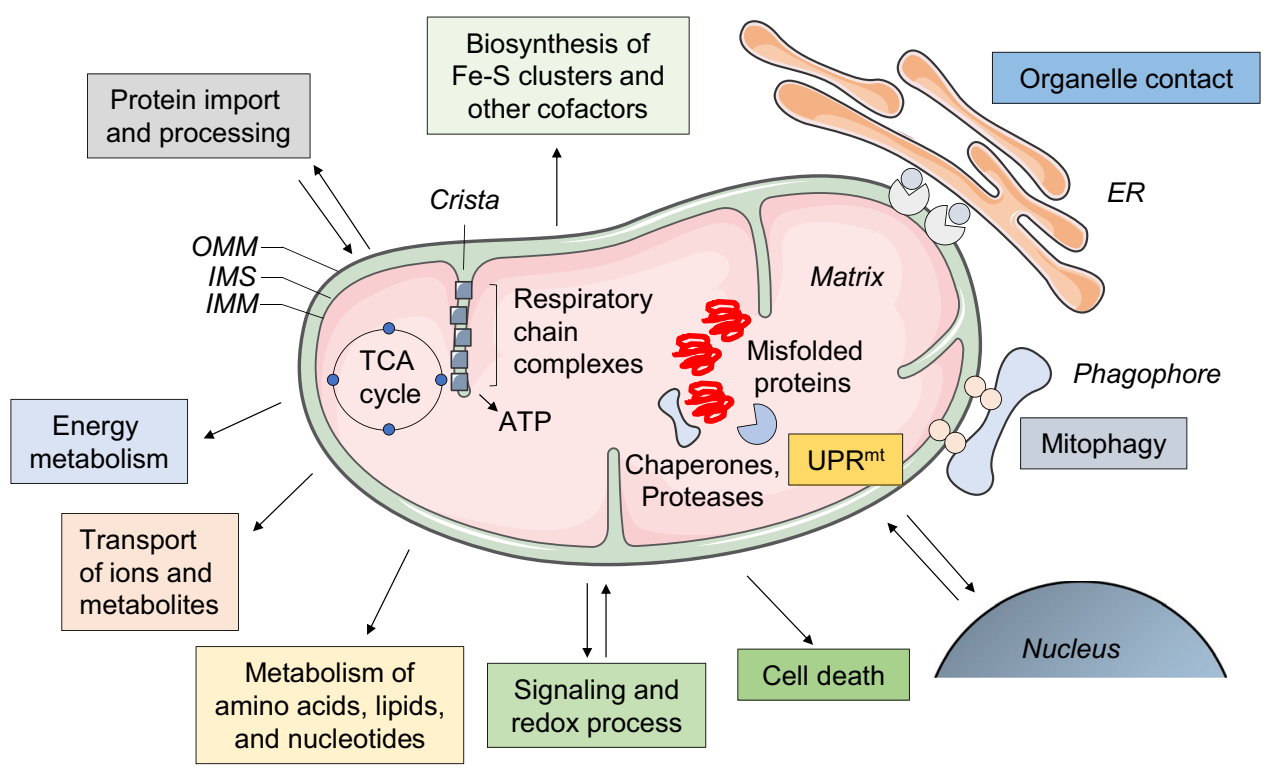

Fig. 1 Mitochondria and their functions. Mitochondria consist of four compartments: outer membrane (OMM), intermembrane space (IMS), inner membrane (IMM), and matrix. Mitochondrial proteins and complexes are involved in energy metabolism with respiration and biosynthesis of ATP; metabolism of amino acids, lipids, and nucleotides; biosynthesis of iron sulfur $(\mathrm{Fe}-\mathrm{S})$ clusters and other

more intimately involved in bioenergetics. The IMM can be further divided into two sub-compartments: the inner boundary membrane and the inner membrane cristae, the latter of which are deeply convoluted invaginations that harbor the machinery required for mitochondrial respiration, including the mitochondrial respiratory chain and $\mathrm{F}_{1} \mathrm{~F}_{0}$-ATP synthase $[22,23]$, ensuring an adequate ATP conversion and energy metabolism (Fig. 1). Altogether, the maintenance of the mitochondrial network structure and integrity is key to performing a wide range of metabolic pathways and signaling cascades that sustain cellular and physiological homeostasis.

\section{Diverse functions of mitochondria}

One of the main functional roles attributed to mitochondria is the ability to coordinate ATP production and energy conversion. Typically, mitochondria comprise the respiratory chain complexes and $\mathrm{F}_{1} \mathrm{~F}_{0}$ ATP synthase, which are located to the inner membrane cristae, and a broad string of nutrient transporters and channels for metabolites and other molecules, which are mostly situated within the matrix and the IMM $[24,25]$. Electrons from the oxidation reactions of metabolites-such as tricarboxylic acid cycle intermediates, amino acids, lipids, and nucleotides - sustain the respiratory chain, ultimately triggering an electrochemical gradient that pumps protons out of the mitochondrial matrix into the intermembrane space [26]. The resulting proton gradient drives cofactors; transport of ions and metabolites; protein import and processing; signaling and redox homeostasis; membrane architecture and dynamics, and interorganelle communication by establishing contact sites; and quality control and degradation processes, and cell death pathways

the conversion of ADP to ATP through $\mathrm{F}_{1} \mathrm{~F}_{0}$ ATP synthase in the process of OXPHOS and enables the transport of precursor proteins and metabolites across the IMM [26]. Under aerobic conditions, this process is responsible for generating the majority of cellular energy and participates in nearly every aspect of metabolic functions.

Beyond its role in energy production and cellular metabolism, the mitochondrial network (Fig. 1) is required for fatty acid, amino acid, and nucleotide metabolism [27], iron-sulfur cluster and cofactor biogenesis [28], and import and processing of precursor proteins that are synthetized on cytosolic ribosomes [29]. Furthermore, mitochondria can serve as signaling platforms that regulate programmed cell death and innate immunity $[12,30]$. For instance, virus infection can activate mitochondrial antiviral signaling proteins, which localize to the OMM and trigger the production of cytokines to counteract infection [31].

Mitochondria also have major roles in sequestering and releasing calcium ions, hence regulating the cellular stores of these versatile signaling molecules. Owing to their intrinsically dynamic nature and the formation of membrane contact sites with the ER, mitochondria utilize calcium transporters $[4,5]$ located at the IMM to control cellular calcium homeostasis in response to contextual signals, ultimately safeguarding a vast array of biological processes and cellular functions. Altogether, the maintenance of a healthy and functional mitochondrial network is particularly critical for the viability of eukaryotic cells as these organelles 
perform essential functions in bioenergetics, metabolism, and signaling.

\section{Mechanisms of mitochondrial quality control}

Mammalian cells have evolved elaborated quality control and surveillance systems to cope with perturbations of homeostasis [32, 33]. For instance, the accumulation of misfolded proteins owing to increased mitochondrial ROS or extra-mitochondrial proteotoxicity or alterations of the respiratory complexes or mitochondrial translation $[34,35] \mathrm{can}$ activate an evolutionarily conserved, transcriptional stress response aptly coined as the mitochondrial unfolded protein response (UPR ${ }^{\mathrm{mt}}$; Fig. 1), part of a larger cell-wide stress response. Within the mitochondria, the $\mathrm{UPR}^{\mathrm{mt}}$ promotes the repair and recovery of mitochondrial network proteostasis, thereby counteracting proteotoxic stress and ensuring the maintenance of mitochondrial and cellular functions [27]. In Caenorhabditis elegans, as part of the UPR ${ }^{\mathrm{mt}}$, an activating transcription factor associated with stress (ATFS-1 [36]), which is normally imported into healthy mitochondria through its mitochondrial protein import sequence and degraded, is instead targeted to the nucleus to trigger adaptive responses that enable the cell to cope with mitochondrial distress [37]. This results in the accumulation of nuclear transcripts that stimulate the recovery of OXPHOS complexes [38, 39], re-establish mitochondrial proteostasis by upregulating chaperones and proteases, and detoxify ROS [40, 41], ultimately restoring mitochondrial proteostasis and homeostasis. However, mammalian studies paint a different picture that implies a process called the integrated stress response, which lowers overall protein production while increasing the abundance of transcriptional factors [40]. Respective findings by Guo et al., [42] and Fessler et al., [43] suggest that mitochondrial dysfunction causes the protein OMA1, which is located on the IMM, to cleave the protein DELE1, a fragment of which enters the cytosol and binds to the enzyme HRI, subsequently activating it. HRI adds a phosphate group to the protein $\operatorname{eIF} 2 \alpha$, and this phosphorylation slows the synthesis of most cellular proteins from messenger RNA, mediated by the ribosome complex, but promotes the production of the transcription factors ATF4, ATF5 and CHOP. Treatments that activate the $\mathrm{UPR}^{\mathrm{mt}}$ have been shown to ameliorate mitochondrial function in amyloid- $\beta$ proteotoxic diseases, such as Alzheimer's disease [44], age-associated amyloidosis [45], and kidney injury [46]. Conversely, tuning the chronic integrated stress response activation might potentially rescue synaptic plasticity loss [47], and reverse age-related cognitive decline [48], and sepsis-induced kidney injury [49].

Recent studies have indicated that similar forms of mitochondrial dysfunction, including damage by paraquat, mtDNA mutations, and misfolded protein accumulation in the matrix, might activate $\mathrm{UPR}^{\mathrm{mt}}$ as well as other quality control and surveillance systems. For example, mitochondrial fusion proteins - such as the dynamin-like mitofusin 1(MFN1), MFN2 and OPA1—combat mitochondrial stress by fusing damaged and/or dysfunctional mitochondria with healthy mitochondria [21,50]. Alternatively, proteins involved in mitochondrial fission-including the cytosolic dynamin-like protein DRP1 and its outer mitochondrial membrane partners-segregate exhausted parts of the mitochondrial network, which are then selectively removed by lysosome-directed degradation pathways [51]. This fascinating interplay between fusion and fission membrane dynamics and mitochondrial quality control mechanisms has been extensively discussed elsewhere [52, 53]. Here, we will emphasize the signaling cascades regulating the mechanisms of mitochondrial elimination by autophagy-lysosome degradation systems.

An essential facet of quality control mechanisms is the activation of (largely selective) degradative cascades that enable cells to dispose of exhausted and/or potentially harmful mitochondria. This may occur through the dynamic and self-regulated process aptly coined as mitochondrial autophagy/mitophagy (Fig. 1), which results in the engulfment of cellular constituents by a double-membrane structure called an autophagosome [33, 54-57]. In functional mitochondria, the phosphatase and tensin homologue (PTEN)-induced kinase 1 (PINK1) is transported into the mitochondrial matrix, where it is processed and cleaved $[52,58,59]$ by matrix processing peptidase (MPP) and presenilins-associated rhomboid-like protein (PARL). As a result, the truncated form of PINK1 is released into the cytosol and degraded by the ubiquitin-proteasome system [60]. Conversely, dissipation of the mitochondrial membrane potential triggers the stabilization of PINK1, facilitating its accumulation on the OMM [61]. This leads to activation of the E3 ubiquitin ligase Parkin, which triggers the ubiquitination of other OMM proteins, culminating in the recruitment of autophagy initiating factors $[60,61]$ and engulfment of damaged mitochondria within autophagy-lysosome degradation pathways.

Beyond its role in organelle quality control and surveillance systems, Parkin has also been found to induce the activation of the transcriptional co-activator peroxisome proliferator-activated receptor gamma co-activator 1 alpha $(\mathrm{PGC} 1 \alpha)$ - a key regulator of mitochondrial biogenesis and energy metabolism - through the ubiquitination of Parkininteracting substrate $[62,63]$. Upon mitochondrial damage, the PINK1-directed recruitment and activation of Parkin at the OMM can trigger the ubiquitin/proteasome system (UPS)-mediated degradation of PARIS-a transcriptional repressor of PGC1-ultimately stimulating mitochondrial biogenesis and function $[62,63]$. In a similar vein, the modulation of PGC1 $\alpha$-elicited mitochondrial biogenesis triggered 
by changes in sirtuin and $\mathrm{NAD}^{+}$metabolism [64], oxidative damage or environmental stress [65, 66], or during exercise [67], seems to trigger PINK1/Parkin-dependent turnover of mitochondria in different tissues and model organisms. Thus, an intimate cross-talk between signaling and degradation pathways safeguards the maintenance of a healthy mitochondrial repertoire, hence preserving its functions in cellular metabolism and organismal homeostasis.

Independent of ubiquitin-driven mitophagy, other OMM-associated mitophagy receptors, such as BNIP3 (BCL2/adenovirus E1B $19 \mathrm{kDa}$ protein-interacting protein 3), NIX (NIP3-like protein X)/BNIP3L, and FUNDC1 (FUN14 domain-containing 1) target damaged and/or dysfunctional mitochondria to autophagosomes by directly promoting the binding of mitochondria with LC3 and $\mathrm{GABA}_{\mathrm{A}}$-receptor-associated protein (GABARAP) through atypical or typical LC3-interacting motifs [68-71]. In a similar vein, cardiolipin - a phospholipid primarily synthesized and distributed along the IMM-may be redistributed to the OMM to interact with LC3-flagged autophagy cargos, initiating a signaling cascade that promotes the engulfment of damaged mitochondria by autophagosomes in response to mitochondrial damage [72], helping preserve cellular and physiological homeostasis.

In contrast to the wholesale mitophagy processes described above, a recently emerging piecemeal mitophagy mechanism of mitochondrial quality control has been described, involving the release of small vesicles excised from the mitochondria and hence aptly termed as mitochondrial-derived vesicles (MDVs [53]). These MDVs have been shown to engulf certain cargos-including outer and inner mitochondrial membrane and/or matrix residing proteinsand transfer them to the lysosome for degradation in an LC3and p62/SQSTM1-dependent manner or to the peroxisome with as yet unclear consequences. Thus, MDVs might act as a first round of defense for mitochondria to remove damaged proteins, preventing the complete failure of the organelle. The diverse repertoire of cellular quality control pathways therefore seeks to ensure the maintenance of a functional mitochondrial network, ultimately sustaining energy metabolism, cellular and organismal homeostasis. Further insights into this fundamental pathway might ultimately lead to new treatments for mitochondrial disorders and other disease entities associated with mitochondrial dysfunction.

\section{Diseases affecting mitochondrial function}

\section{Primary and secondary mitochondrial disorders}

Given the crucial roles of the mitochondrial network in cellular energy production and homeostasis, it is not surprising that disorders which disrupt mitochondrial function are of grave consequence to the individual. When inherited, these disorders are often classified as primary or secondary mitochondrial diseases. Primary mitochondrial diseases include inherited disorders that disrupt OXPHOS or mitochondrial structure and function, such as abnormalities in the production of cofactors and vitamins, or other alterations in the TCA cycle and pyruvate metabolism [73]. Of the 1500 proteins estimated to participate in mitochondrial function and maintenance, nearly 400 have been reported to cause primary mitochondrial disease [74], including all 37 mitochondrial encoded genes. Altogether, primary mitochondrial disorders affect approximately 1:4300 births [75].

The heterogeneity of the processes disturbed by primary mitochondrial disorders is reflected by the phenotypic variability of the patients, in terms of tissues and organs affected, as well as by age of onset and presenting symptoms [73, 74]. Patients may exhibit manifestations in almost any tissue or organ, in a multi-systemic or tissue-specific manner, from the first days to weeks of life until after several decades [76]. Nevertheless, along with lactic acidosis, frequently identified symptoms involve encephalopathy, cardiomyopathy, renal insufficiency, and liver failure [73, 74] (Fig. 2). Clinical features in these disorders have been expertly reviewed elsewhere [74, 77].

A considerable body of evidence suggests that the mitochondrial stress responses triggered by a primary molecular defect in the organelle, and not defects of OXPHOS per se, are the major contributing factor to the clinical and biochemical features of mitochondrial disorders [3]. Such mechanisms, including the $\mathrm{UPR}^{\mathrm{mt}}$, are likely to be activated by secondary mitochondrial diseases-i.e., disorders of genes/ proteins not directly involved in OXPHOS or mitochondrial integrity, but which may indirectly compromise these essential processes through toxic metabolites or missing products.

A prototypical group of secondary mitochondrial disorders are the organic acidurias, a collection of diseases including branched-chain ketoaciduria, isovaleric aciduria, propionic aciduria and methylmalonic aciduria (MMA), whose disrupted pathways take place within the mitochondria [78]. In patients with these disorders, deficient activity of enzymes involved in energy producing, catabolic pathways, result in the accumulation of mono-, di- or tricarboxylic acids in the brain and other tissues as well as in the urine, plasma, and cerebrospinal fluid. Although the enzymes primarily disturbed in organic acidurias are not directly involved in OXPHOS, they do affect mitochondrial energy metabolism and mitochondrial homeostasis, whose dysfunction seems to have a major role in disease development [79]. This can also be seen from the clinical manifestations of these disorders, whereby frequent clinical symptoms include cardiomyopathy, optic atrophy and basal ganglia abnormalities, liver dysfunction and kidney failure, in analogy to primary mitochondrial disorders. All 
Fig. 2 Comparison of the clinical presentation of primary mitochondrial diseases and MMA. Note the overlap of most clinical features, with some interesting exceptions, including cardio/myopathy (rare in MMA)

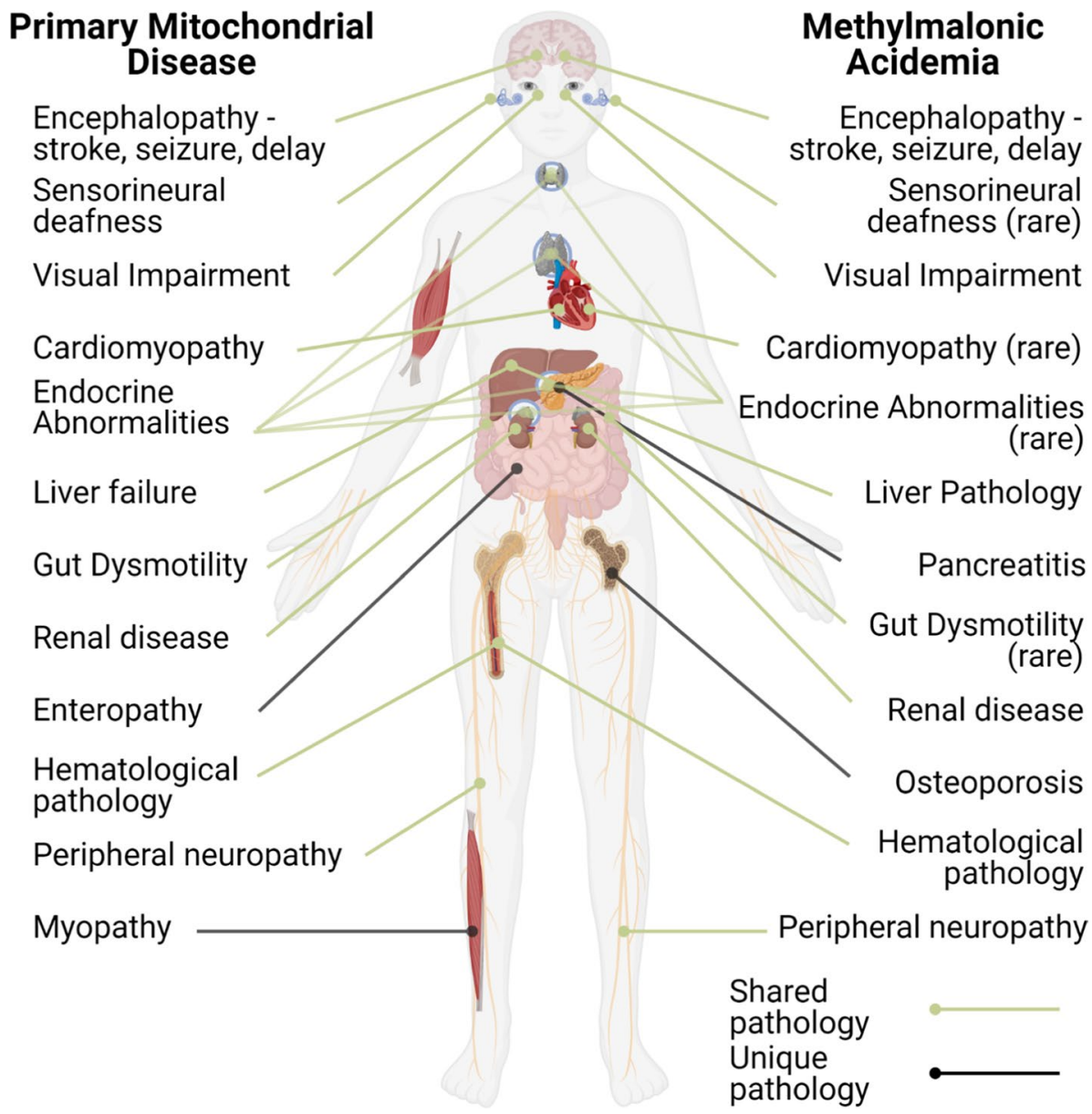

these manifestations, to varying degrees, have been found in MMA (Fig. 2), which can, therefore, be used as a prototypical model of (secondary) mitochondrial disease.

\section{MMA is a paradigm organic aciduria with secondary mitochondrial disease}

\section{Gene, mutations, and protein functions}

MMA can be caused by a series of defects, most of which lead to deficiency of the enzyme methylmalonyl-CoA mutase (MMUT), with a combined prevalence estimated to be 1:50,000 [80]. Mutations in the MMUT gene account for approximately $50 \%$ of all cases of isolated MMA, and to date almost 300 inherited mutations have been described in $M M U T$, the majority of which are of the missense type and associated with the more severe phenotype that manifest in the absence of enzyme activity $\left(\right.$ mut $\left.^{0}\right)$ [81]. Nevertheless, most mutations are private, and only a few mutations occur in several patients, including c.655A $>$ T (p.Asn219Tyr), c.1106G > A (p.Arg369His), c.2080C > T (p.Arg694Trp) and c. $2150 \mathrm{G}>\mathrm{T}$ (p.Gly717Val) $[81,82]$.
MMUT catalyzes the reversible isomerization of L-methylmalonyl-CoA to succinyl-CoA - an intermediate of the tricarboxylic acid cycle that is further processed by succinyl-CoA ligase. This represents the culmination of propionyl-CoA catabolism, most of which derives from the breakdown of branched-chain amino acids and odd-chain fatty acids, although a significant proportion of propionylCoA may be derived from gut bacteria [83]. Propionyl-CoA is converted to D-methylmalonyl-CoA by the action of propionyl-CoA carboxylase, defects in which cause propionic aciduria - a disease that phenotypically and biochemically overlaps with MMA [84]. For use by MMUT, D-methylmalonyl-CoA is racemized to the $\mathrm{L}$ configuration by methylmalonyl-CoA epimerase (MCEE). For proper function, MMUT requires the vitamin $\mathrm{B}_{12}$ derived cofactor adenosylcobalamin. Therefore, defects in the genes $M M A A$ [85], $M M A B$ [86] and $M M A D H C$ [87], which are responsible for the synthesis or disposition of adenosylcobalamin, may also lead to MMUT deficiency and therefore MMA, while biochemically mild MMA can be associated with mutations in MCEE [88, 89], succinyl-CoA ligase (SUCLG1 or SUCLA2) [90] or ACSF3 (combined malonic and methylmalonic aciduria) [91, 92]. 


\section{Healthy mitochondrion}
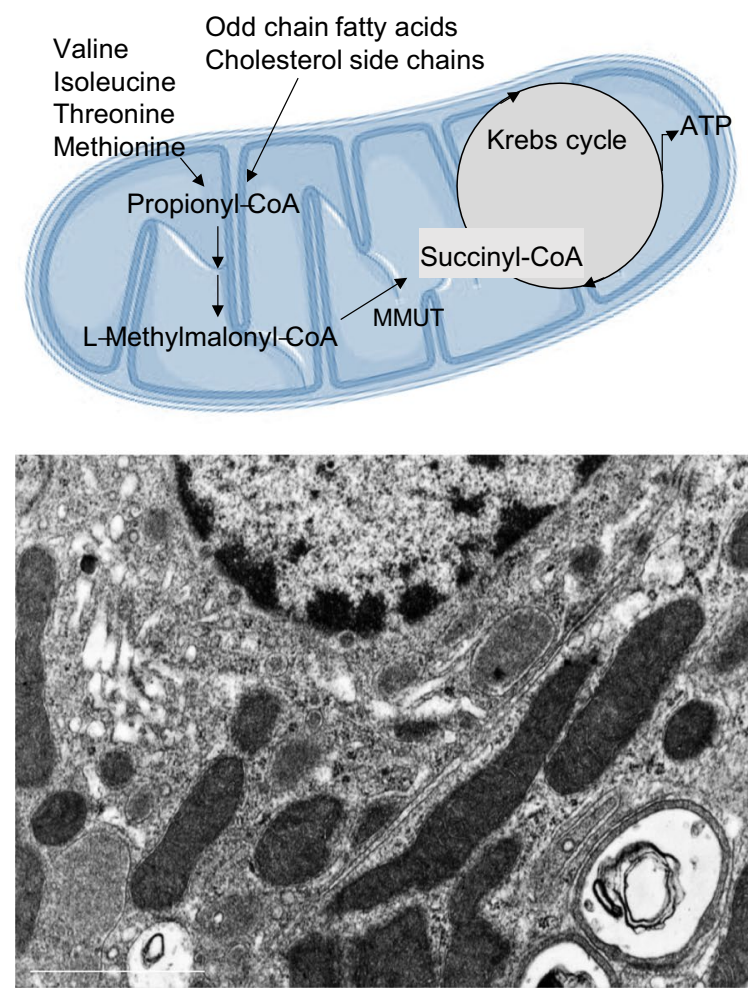

Fig. 3 Loss of the enzyme MMUT leads to accumulation of organic acids and mitochondrial abnormalities. Inactivating mutations in the MMUT gene encoding the mitochondrial enzyme methylmalonyl-coenzyme A mutase, which mediates the terminal step of branched-chain amino acid and odd-chain lipid catabolism, results in the accumulation of metabolites (i.e., methylmalonic acid, propionic acid, and 2-methylcitric acid) and lack of anaplerosis. This triggers

In MMA, organic acids in addition to the substrate methylmalonyl-CoA accumulate (Fig. 3), leading to secondary inhibition of several enzymes in TCA cycle and respiratory chain metabolism, the urea cycle, and mitochondrial transport [93]. This impairs mitochondrial respiration and ammonia metabolism, pathologies shared by other organic acidurias and primary mitochondrial disorders [94]. All of these are likely to play a role in the clinical presentation of this disease.

\section{Clinical features}

The clinical presentation of MMA is often nonspecific and patients may present with acute or chronic symptoms at any age [84]. Nevertheless, a classical early-onset presentation in the short term may include metabolic decompensation with vomiting, feeding problems, metabolic acidosis and hyperammonemia with neurological deterioration including muscular hypotonia, irritability and lethargy, which in the most severe cases may lead to coma and death [95].
Methylmalonic acidemia mitochondrion
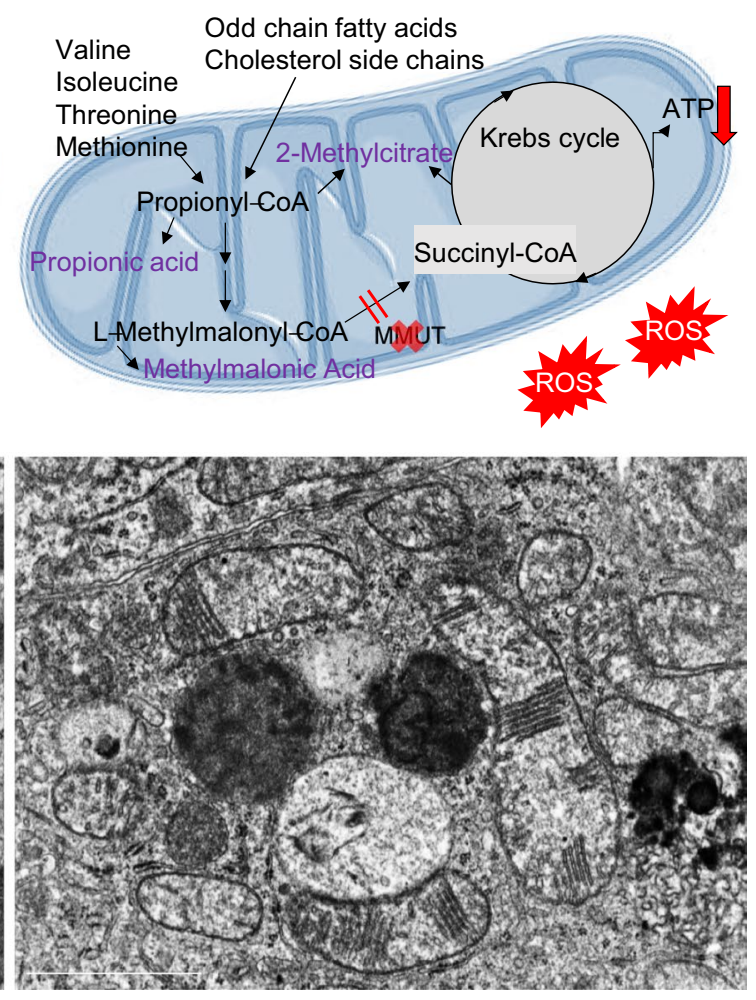

ultrastructural (i.e., presence of abnormal mitochondria with disorganized cristae) and/or functional (i.e., impaired mitochondrial energetics and redox homeostasis) mitochondrial alterations, ultimately causing severe organ dysfunctions that primarily affect brain, liver, and kidney. Electron micrographs courtesy of Francesca Diomedi (Adapted from Luciani et al. [130])

Current treatments, including intensive care, dietary modulation (protein restriction, precursor free amino acid supplements, tube-feeding) and pharmacotherapy (administration of cobalamin, carnitine and reduction of intestinal flora with antibiotics), have resulted in many patients surviving these initial crises [96, 97]. However, affected patients remain vulnerable to life-threatening metabolic decompensations, often show only slight improvement, and are not protected from long-term complications [96, 98]. The most striking longterm complications involve neurological symptoms and kidney manifestations $[96,99,100]$; while cardiomyopathy, a common feature of propionic aciduria $[84,101]$ and primary mitochondrial disorders, is not frequently found in MMA.

\section{Brain abnormalities}

Neurological impairment in MMA can take many forms. In 52 children with MMA, the most common computed tomography (CT) and magnetic resonance (MR) brain sectional imaging findings were ventricular dilation (17 patients), 
cortical atrophy (15), periventricular white matter abnormality (12), thinning of the corpus callosum (8), subcortical white matter abnormality (6), cerebellar atrophy (4), basal ganglia calcification (3), and myelination delay (3) [102]. However, the clinical significance of many of these radiological findings is often unclear.

Neuropathological findings in white matter tracts, cerebral and cerebellar cortex are typically isolated to atrophy, reactive gliosis, spongiosis and hypomyelination [102-104]. In MMA, periventricular white matter abnormalities, which are defined as delayed myelination, occur early and may be partially reversible. Cortical atrophy in MMA is defined as ventricle and sulci widening and as with delayed myelination, early detection and treatment (e.g., before 2 years of age) may result in normalization after a period of time [102-106]. Seizures are also a recognized symptom of MMA which may be present at presentation or appear later [84, 96, 107].

Neurological impairment most commonly manifests as movement disorders, such as involuntary tremor, gait instability, dystonia, hypotonia, muscular rigidity and chorea [108]. These are often associated with "metabolic stroke", which leads to bilateral focal brain lesions, characteristically in the globus pallidus externa and other regions of the basal ganglia, which occur in the absence of vascular stroke aetiologies [104, 106, 109-111]. Necrotic lesions in the basal ganglia often occur during or shortly after metabolic decompensation [84]. Nevertheless, in some reported cases, necrosis and CT/MR findings in patients do not occur with a history of severe metabolic acidosis [105]. Whilst the picture around the susceptibility of the basal ganglia is not fully illuminated, findings of bilateral lesions are consistent throughout differential diagnosis of organic acidurias and primary mitochondrial diseases. This may be related to the fact that basal ganglia and particularly the putamen and globus pallidus have a rich vasculature supply (middle cerebral artery, branching to lenticulostriate arteries) [112-114], an abundance of mitochondria [115-117] and the network as a whole is reported to demonstrate high metabolic activity and increased glucose and oxygen utilization [118-120]. Consistently, lacunar infarcts in the pars reticulata of the substantia nigra, a region functionally and histologically identical to the globus pallidus interna, have also been found in patients with MMA, supporting the idea that specific celltypes show particular susceptibility [121].

Finally, long-term neurological damage can be present in the visual and auditory systems. Especially, optic atrophy is an increasingly recognized late presentation of MMA, resulting in acute or chronic visual loss [107, 122]. Optic atrophy in MMA has been hypothesized to arise from mitochondrial dysfunction, due to the similarities in age at onset, presentation and progression between MMA and primary mitochondrial disorders, as well as the apparent beneficial effect of supplementation with coenzyme Q10 and $\alpha$-tocopherol [79]. Sensorineural hearing loss is rare, with yet unclear etiology.

\section{Kidney damage}

Kidney specific defects manifest as tubulointerstitial nephritis and renal tubular acidosis. End stage renal disease may also occur as early as the second decade of life. Most patients have evidence of mild tubular dysfunction during childhood, usually without overt signs of renal Fanconi syndrome, which may become very profound during episodes of metabolic decompensation that are usually triggered by infection, ultimately causing renal salt and bicarbonate losses. A study examining a cohort of 30 French patients found chronic kidney disease in $47 \%$ with a median onset of 6.8 years [123]. Similar results have been reported in a large cohort of 273 European patients, whose clinical evolution was related to the type of defect [124]. Proteinuria and hematuria are usually absent in most patients, and renal histopathology shows signs of tubulointerstitial nephritis both in humans and in animal models of MMA [125, 126]. Renal ultrasound usually shows poorly differentiated kidneys with decreased growth overtime [127]. The mechanisms of cellular toxicity, caused by accumulating organic acids and the mitochondrial defects, and its implication in the pathogenesis of kidney damage in MMA, are discussed in the section below.

\section{Insights into mitochondrial disease pathways - the role of impaired mitophagy}

Complete $\left(m u t^{0}\right)$ or partial $\left(m u t^{-}\right)$loss of the enzyme MMUT leads to the accumulation of toxic organic acids (e.g., methylmalonic acid, propionic acid and 2-methylcitric acid) and loss of anaplerosis (Fig. 3, top panel). This presumably trigger the structural and functional abnormalities in the mitochondrial network (Fig. 3, bottom panel) which likely drive severe organ dysfunction affecting primarily brain and kidney. Reinforcing this concept, the metabolic signature of high urinary excretion of toxic organic acids, as well as mitochondrial morphological abnormalities, dysregulation of the respiratory chain complex, causing decreases in mitochondrial energy production, and augmented production of reactive oxygen species and oxidative damage have been described in kidney cells [126] and biopsies from MMA patients [128, 129]. However, mechanistically, how the enzyme deficiency begets mitochondrial distress and cell toxicity has remained incompletely understood.

Prolonged or unrepairable damage can lead to elimination of mitochondria through a selective autophagic process termed mitophagy. Given the accumulation of MMA-damaged mitochondria and autophagic vesicles, MMUT deficiency might compromise PINK1/Parkindirected priming of MMA stressed mitochondria to 
autophagic-lysosomal degradation. Using the translocation of the protein parkin RBR E3 ubiquitin protein ligase (PRKN) to mitochondria as a bona fide reporter of PINK1-PRKN-dependent priming mechanisms, MMA patient-derived kidney cells display a decrease in numbers of PRKN ${ }^{+}$clusters and translocation of PRKN to damaged mitochondria, in both normal and stress-induced conditions [130-132]. As a direct consequence of defective PINK1-PRKN priming mechanisms, mutant cells fail to deliver their damaged mitochondria to autophagy-lysosome degradation systems, causing the accumulation of dysfunctional mitochondria that trigger cellular distress and kidney damage [130-132]. Expression of the wildtype PINK1 in MMA patient-derived kidney cells activates mitophagy-mediated degradation of diseased mitochondria, thereby averting mitochondria-derived epithelial distress and cell damage. These findings suggest that anomalies in PINK1/Parkin-mediated quality control and surveillance systems might thus intersect the mitochondrial alterations induced by MMUT deficiency to reach a high level of mitochondrial dysfunction that ultimately contributes to the pathogenesis in MMA patients (Fig. 4). The concept that defective mitochondrial autophagy/ mitophagy and uncontrolled cellular stress might contribute to the MMA disease is in line with the observed correlation between mitochondrial dysfunction, oxidative stress, and circulating lipocalin-2 (LCN2) - a secreted iron-transporting protein produced by kidney tubules following cellular damage in a cohort of patients with MMA [126, 129].
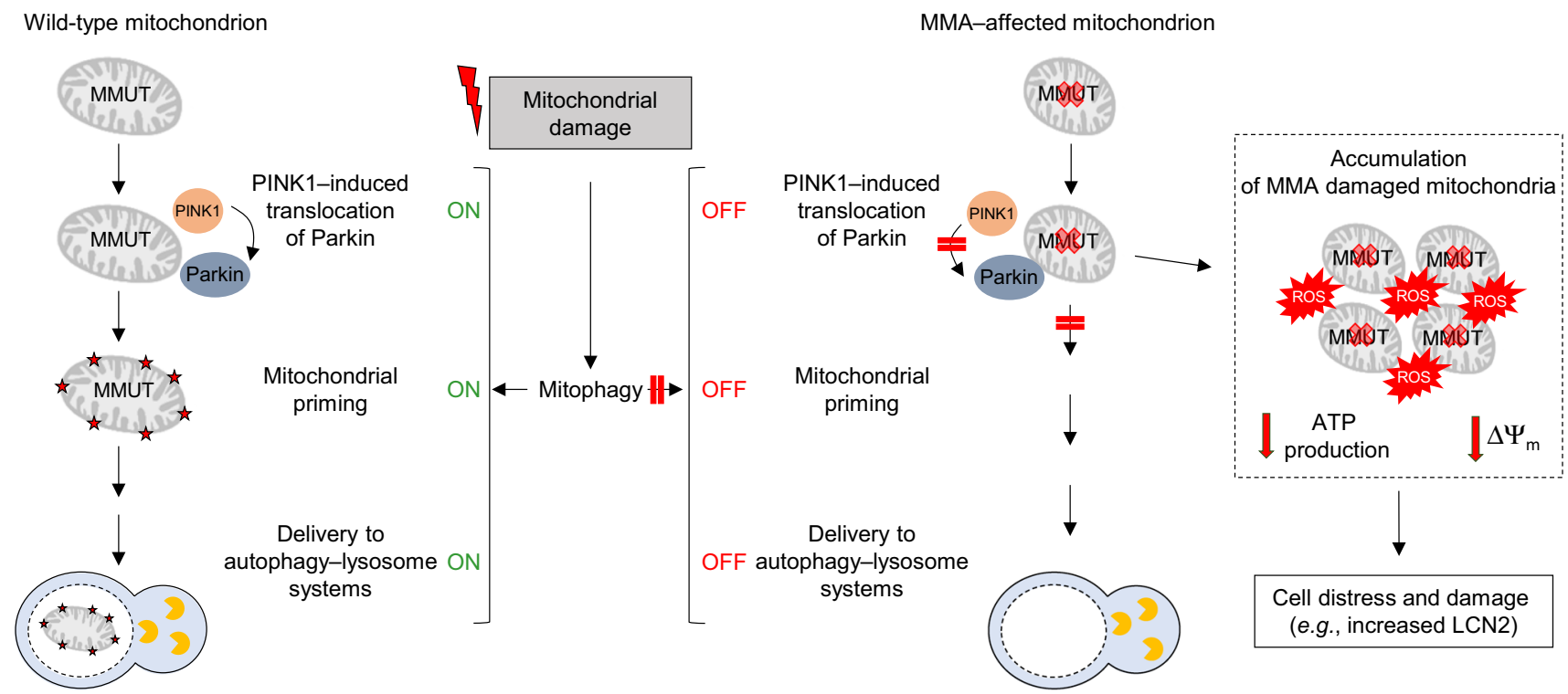

Fig. 4 Model depicting the link between MMUT, mitochondria, organelle quality control and surveillance systems, and epithelial homeostasis in normal and MMA-affected kidney cells. In MMA-affected kidney cells and zebrafish, deficiency of the enzyme MMUT and the resulting accumulation of toxic organic acids trigger mitochondrial alterations that are characterized by a collapse of the mitochondrial membrane potential $(\Delta \Psi \mathrm{m})$, abnormal bioenergetics profiling, and increased generation of mitochondrial ROS and oxidative stress. Faulty execution of PINK1-Parkin-mediated mitophagy

induced by MMUT deficiency impedes the delivery of damaged mitochondria and their dismantling by autophagy-lysosome degradation systems. This leads to accumulation of damaged and/or dysfunctional, ROS-overproducing mitochondria that ultimately trigger cellular distress (as evidenced by Lcn2 overproduction) in patient-derived cells and disease-relevant phenotypes (as testified by liver/kidney mitochondriopathy, behavioral abnormalities and an excess of mortality) in mmut-deficient zebrafish (Adapted from Luciani and Devuyst [131]) 


\section{Cell and animal-based disease models of MMA}

\section{Patient-derived cells and tissue culture}

Assessments of the mode of action of protein-damaging mutations require proof-of-concept studies in physiologically relevant cell culture based-systems. Traditional cell-based models typically rely on cancer-derived or immortalized cells that fail to adequately recapitulate the complex features of the in vivo disease phenotype [133]. The use of patient-derived primary cell samples aims thus to overwhelm the disadvantages and limitations of using transformed cell lines for dissecting disease mechanisms. These samples also provide more clinically relevant cellbased models for systematically testing drug discovery and screening pipelines or identifying novel drug-repositioning opportunities.

A common use of patient-derived cell lines in MMA is that of primary skin-derived fibroblasts for disease diagnosis. Although not a clinically important cell-type, fibroblasts express all proteins relevant to MMA. As such, they are routinely utilized for activity analysis of individual enzymes, particularly MMUT, or diagnosis of defects in the propionate pathway through incorporation of radiolabeled propionate into purified proteins [134]. To assess disease mechanisms, fibroblasts have been subjected to proteomics and metabolomics analysis, whereby dysregulation of serine metabolism was found [135]. A potentially more exciting use of patient-derived fibroblasts involves converting them to induced pluripotent stem cells (iPSCs), which can subsequently be differentiated into all cell types of the human body. At least two MMA patient-derived iPSC lines have been generated [136]. Differentiation of these, particularly into neural cells, may provide novel insights into CNS disease mechanisms, which are currently poorly understood.

Other potentially relevant patient-derived cell types are primary hepatocytes. Since the liver is a site for some clinical manifestations, including hepatomegaly [79] and potentially more concerning hepatocellular carcinoma [137] hepatocytes may be an important cell type to investigate. Using a novel organotypic system which utilizes hemodynamic flow, Collado and colleagues [138] demonstrated accumulation of disease relevant metabolites in patient-derived primary hepatocytes.

As MMUT is robustly expressed within the mitochondria of kidney tubular cells [130], we analyzed the properties of the mitochondrial network in kidney tubular cells derived from the urine of either healthy controls or $m u t^{0}$ MMA patients harboring inactivating mutations in $M M U T$ (henceforward referred to as MMA cells). These studies revealed that MMUT deficiency causes accumulation of damaged and dysfunctional mitochondria, ultimately triggering cell distress and kidney damage $[128,130]$. These dysfunctions are associated with an exaggerated production of LCN2-a small iron-transporting protein associated with kidney disease progression [139] and metabolic disease [140]. In line with recent studies stating dysregulation of autophagy-lysosome degradation pathways in mitochondria-related diseases [141] and in tissue samples from patients with MMA [129], autophagosome-associated LC3-II form, punctate LC3-positive structures, and electron microscopy structures compatible with autophagic vacuoles accumulate in MMA cells compared to their corresponding controls. Furthermore, MMA cells display decreased mitochondrial membrane potential and mitochondrial bioenergetics. These alterations are paralleled by a major mitochondrial oxidative stress $[128,130]$, as testified by the elevated production of mitochondriaderived ROS (MitoSOX, a live-cell permeant indicator of mitochondrial ROS), which are observed also in other mitochondria-related diseases [126, 141]. Small-molecules targeting oxidative stress have been shown to be efficacious in repairing mitochondrial homeostasis and function in MMA cells [130], demonstrating the utility of these cellular systems for translating disease knowledge to clinical outcomes for therapeutic approaches.

\section{Animal models}

The availability of animal-based models represent a fundamental opportunity not only to gain insights into signaling frameworks driving the pathogenesis of rare genetic diseases but also to test potential therapeutic strategies.

Seven different mouse models of MMA have been generated to date, each with their own strengths. Two knock-out models of Mmut have been derived, resulting in complete null mutants [142, 143]. These mice displayed massive elevations of disease related metabolites, however, most pups did not survive past the first 24-48 h. Backcrossing to a mixed background did allow a small fraction of animals to survive the neonatal period [125]. These animals showed megamitochondria in hepatocytes, proximal tubule cells and exocrine pancreas, and displayed a clinical phenotype of tubulointerstitial renal disease. Ion-abrasion scanning electron microscopy from the liver of 4 day old Mmut knockout mice confirmed the beginning of structural changes, but before conversion to megamitochondria [144]. Partial rescue was mediated by creating transgenic mice hemizygous for a human wild-type MMUT transgene [145] but not one incorporating p.Arg403* [146]. These mice were smaller than their littermates and had elevated methylmalonic acid in urine, plasma, and tissues. Full rescue has been achieved through expression of a stable transgene under the control 


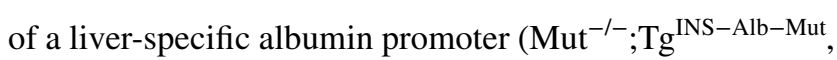
[126]) and a skeletal muscle specific murine creatine kinase promoter ( $\left.\mathrm{Mut}^{-/-} ; \mathrm{Tg}^{\mathrm{INS}-\mathrm{MCK}-\mathrm{Mut}},[129]\right)$. Nevertheless, $\mathrm{Mut}^{-/-} ; \mathrm{Tg}^{\text {INS-Alb-Mut }}$ mice displayed chronic tubulointerstitial nephritis, had ultrastructural changes in the proximal tubule mitochondria, increased expression of Lcn2 [126] and severe kidney disease [147] while $\mathrm{Mmut}^{-/-} ; \mathrm{Tg}^{\text {INS-MCK-Mut }}$ mice showed growth retardation, and liver and kidney mitochondriopathy [129]. A knock-in mouse [148] has been produced by Forny and colleagues which incorporated a missense change (p.Met698Lys in mouse) corresponding to a known patient mutation (p.Met700Lys in human). When present in the hemizygous form, along with a knock-out mutation on the second allele, these mice show a mild MMA phenotype which nevertheless includes elevated metabolites (methylmalonic acid, propionylcarnitine/acetylcarnitine ratio) early signs of renal dysfunction and TCA cycle alterations in liver mitochondria [149]. These symptoms are exacerbated in the presence of a high-protein diet, whereby mice exhibited a strong growth defect, morphological changes in the liver, low bone mineral density and ovarian atrophy [150]. Surprisingly, however, fasting for 24 or $48 \mathrm{~h}$ resulted in reduced metabolite levels [151].

A transgenic zebrafish model of MMA has been generated by using CRISPR/Cas9 genome editing [130]. The obtained zebrafish mutant line carries an 11-bp-CRISPR/ Cas9-induced deletion ( $\left.m m u t^{\text {del11/de111 }}\right)$, generating a premature stop codon within exon 3, resulting in a truncated protein deprived of its catalytic activity. Analogous to metabolic and mitochondrial alterations reported in MMA patient-derived kidney cells, both liver and kidney of mmut-deficient zebrafish accumulate methylmalonic acid and display altered mitochondrial morphology characterized by increased mitochondrial circularity with perturbed cristae organization. Metabolic flux analyses have revealed impaired mitochondrial bioenergetics in mmut-deficient zebrafish when compared to control larvae. These changes are paralleled by a major mitochondrial oxidative stress, suggesting an evolutionary conservation of this connection. Mutant zebrafish faithfully recapitulate MMA characteristic disease-relevant phenotypes, such as liver mitochondriopathy, behavioral abnormalities, and an excess of mortality. These phenotypes are rescued by feeding the mutant zebrafish with a low protein diet-a current supportive care strategy used in MMA patients [84]. Intriguingly, these findings revealed that restoring mmut activity in the liver, which normalizes the levels of methylmalonic acid metabolite and blunts excessive mortality, does not protect the mmut-deficient zebrafish from the abnormal swimming phenotype. The latter observation suggests that mmut-induced mitotoxicity in other cell types and organs (i.e., central nervous system, optic nerve and/or muscle) might govern the phenotypes encountered in mmut-deficient zebrafish. Given the faithful recapitulation of disease phenotypes associated with MMA, and the recent technological advances in scale, time, and cost, and multiplexing of conditions and the potential of automation, zebrafish might thus serve as a forefront tool for chemical phenotypic screens of "first in class" drugs, ultimately translating promising preclinical drug candidates into clinical success.

\section{Current therapies and future targetable strategies}

Isolated MMA makes up one of the most frequent groups of inborn errors of metabolism, which often manifest in early childhood and are associated with high morbidity and mortality. There are no curative treatments for MMA. The available therapeutic approaches, such as dialysis and intravenous glucose infusion in the acute situation, as well as dietary protein restriction, supplementation with carnitine and vitamin $B_{12}$ and potentially liver and/or kidney transplantation as long-term measures, aim for metabolic stabilization of the patient $[84,97]$. While these measures can substantially decrease mortality and the overall morbidity, they cannot completely prevent long-term complications. Therefore, there is an urgent need to yield promising targetable interventions in the early course of MMA.

Previous studies showed that kidney dysfunction and levels of circulating Lcn 2 could be abrogated in a transgenic mouse model of MMA by administrating ubiquinone, a bioavailable form of coenzyme $\mathrm{Q}_{10}$ that acts on mitochondria, and vitamin E [126]. Taking advantage of a drug-disease network-based computational modeling approach (Mantra 2.0; Mode of Action by Network Analysis; http://mantra.tigem.it), we identified new drugable pathways that might potentially counteract cellular dysfunctions associated with MMA [130]. These in silico analyses indicated that targeting mitochondrial function and redox homeostasis might reverse disease phenotypes associated with MMUT deficiency. In line with these predictions, treatment of MMA cells with mitochondriatargeted antioxidants such as mito-TEMPO partially rescues mitochondrial network homeostasis and improves its function, blunts any increases in mitochondrial ROS and autophagy markers, and prevents epithelial distress and cell damage [130]. In a similar vein, treatment with low doses of MitoQ, another well-known mitochondriatargeted antioxidant, alleviates the mitochondrial oxidative stress, improves behavioral phenotypes, and reduces the excessive mortality in the mmut-deficient zebrafish model of MMA [130]. Importantly, both pharmacological interventions did not modify the levels of MMA metabolite in either MMA cells or mmut-deficient zebrafish, supporting the concept that mitochondrial targeting acts independently of the elevation of toxic MMA metabolites. Ultimately, regimens that enhance PINK1-Parkin-directed 
mitophagy and mitochondrial quality control might facilitate the degradation of MMA-affected mitochondria and attenuate the cellular and metabolic alterations that drive life-threatening systemic manifestations in MMA patients. In this case, the administration of either synthetic or natural chemical compounds with direct or indirect mitophagyaugmenting properties [e.g., $\mathrm{NAD}^{+}$precursors, such as nicotinamide riboside (NR), nicotinamide mononucleotide (NMN) and nicotinamide (NAM) (reviewed in [11]); autophagy and mitophagy activators such as spermidine [152], resveratrol [153], urolithin A [34]; mitochondrial stress response inducers such as actinonin [154] and doxycycline [44] would thus constitute an attractive (and potentially feasible) strategy for therapeutically treating this devastating disorder.

Alternatively, using primary hepatocyte disease models, a group of scientists from HemoShear Therapeutics have shown that supplementation with disodium citrate partially rebalances the concentration of TCA cycle intermediates [138], while supplementation with a short-chain carboxylic acid (2,2-dimethylbutanoic acid) is able to reduce intracellular concentrations of metabolites related to propionyl-CoA and methylmalonyl-CoA [155, 156]. This latter molecule is now part of a therapeutic clinical trial (NCT04732429) for MMA.

Beyond promising candidate targets for drug development, cell-based therapy including the direct replacement of defective MMUT through the administration of gene or mRNA therapy has been tested in various mouse models of MMA. In a first attempt, adenovirus mediated gene therapy of Mmut under the control of a cytomegalovirus promoter delivered either intramuscularly or intrahepatic demonstrated that half of the Mmut knock-out mice provided with intrahepatic injections, which would otherwise die within $48 \mathrm{~h}$ postnatal, survived past weaning [157]. Later, treatment with adeno-associated virus (AAV) serotype-8 resulted in much longer survival of Mmut-KO mice [158, 159], while injection with Mmut under a liver-specific thyroxine-binding globulin promoter resulted long-term phenotypic correction following administration with AAV8 [160], or AAV9 [161]. Further liver directed therapies have included lentiviral administration of a codon optimized human MMUT transgene, which resulted in correction of the growth defect, and reduced methylmalonic acid in plasma, urine and tissues [162]. Most recently, a promoterless $M M U T$ transgene has been delivered into the albumin locus by AAV, resulting in improved animal survival and an apparent growth advantage to correct hepatocytes [163]. Finally, using non-viral delivery, lipid nanoparticle enclosed human $M M U T$ mRNA delivered via tail vein injection and targeting the liver has improved survival and weight gain and reduced circulating metabolites in $\mathrm{Mmut}^{-/-} ; \mathrm{Tg}^{\mathrm{INS}-\mathrm{MCK}-\mathrm{Mut}}$ and $\mathrm{Mmut}^{-{ }^{-}} ; \mathrm{Tg}^{\mathrm{INS}-\mathrm{CBA}-\mathrm{G} 715 \mathrm{~V}}$ mice $[164,165]$. Both AAV-mediated gene therapy using promoterless MMUT (NCT04581785) and LNP-mediated mRNA therapy (NCT03810690) are the focus of ongoing phase $1 / 2$ clinical trials.

\section{Conclusions}

The maintenance of a healthy mitochondrial network is particularly crucial for cellular and organismal homeostasis, and loss-of-function mutations that impair the function of mitochondria can invariably confer a potentially devastating vulnerability to many different cell types, ultimately contributing to a broad spectrum of diseases. Inherited defects in mitochondrial-localized proteins and/or enzymes, as exemplified here by MMA, might disable PINK1/Parkin-mediated quality control and surveillance systems, triggering a level of mitochondrial dysfunction that drives cellular distress and tissue damage.

The mitochondrial dysfunction and impaired mitophagy flux seem to play a pivotal role in the pathogenesis of kidney damage, a so far poorly understood process, and, possibly, also in the generation of central nervous system-related symptoms and of neuropathological signs in MMA patients. Interestingly, and unlike cardiomyocytes of rodents lacking Pink1 [166] or Parkin [167], which display accumulation of morphologically abnormal mitochondria and heart pathology, patients with MMA rarely manifest symptoms of cardiomyopathy [84, 101]. The reasons for this exquisite context and cell type specificity are fundamentally enigmatic. Tentative hypotheses, which warrant further investigations in non-affected tissues, include the existence of compensatory (stress-evoked) surveillance systems that coordinate the mitochondrial turnover and quality control, or the cumulative effect of the metabolic perturbations resulting from the absence of MMUT enzyme and the loss of PINK1/Parkin-mediated mitophagy to reach a high level of mitochondrial dysfunction that ultimately causes disease relevant-phenotypes in MMA. Indeed, the accumulation of methylmalonic acid in the blood of older people seems to sustain age-induced spread of cancer [168]—an emerging complication encountered in patients with MMA [169].

The mechanisms by which MMUT deficiency suppresses PINK1/Parkin-directed "eat me" signals and hence mitophagy, remain equally elusive. An intriguing scenario might be that MMUT deficiency might alter the stability of PINK1 by disabling the interaction with yet-to-be defined factors that protect PINK1 from processing and degradation. Alternatively, MMUT deficiency might trigger stress-related posttranslational modifications such as S-nitrosylation that inhibits PINK1 kinase activity and hence mitophagy-directed degradation pathways [168]. We suspect that dysregulation of adaptive response to mitochondrial stress might also contribute to maladaptation and disease in patients with MMA, 
and this will require further studies to understand the effects of MMUT mutations on mitochondrial repair pathways, such as UPR ${ }^{\mathrm{mt}}$ and mitochondrial biogenesis. These questions are just examples of all the exciting work that lies ahead to comprehensively dissect the cell type-specific functions of MMUT and mitochondrial quality control systems. A current challenge is to translate the knowledge gained from fundamental studies of mitochondrion biology to the treatment of MMA and other mitochondria-related diseases. In this regard, the use of informative preclinical models and physiologically relevant cellular systems, coupled with improved knowledge of cell biology-disease signatures and the recent advances in multi-omics technologies, might accelerate the development of therapeutics that can halt the progression of MMA disease as well as other rare and more common diseases associated with mitochondrial dysfunction.

Author contributions AL and DSF conceptualized and wrote the article with inputs and comments from all the authors; VS critically edited and revised the work; MCSD contributed to research data for the article, to the discussion of the article's content, and generated Fig. 2; LPG generated Fig. 1.

Funding Open Access funding provided by Universität Zürich. A.L. is supported by Cystinosis Research Foundation (Irvine, CA, USA) and the Swiss National Centre of Competence in Research (NCCR) Kidney Control of Homeostasis (Kidney.CH). D.S.F. is supported by the Swiss National Science Foundation (310030_192505). M.D. is supported by the Swiss National Science Foundation (310030 175779). A.L. and D.S.F. received financial support from the University Research Priority Program of the University of Zurich (URPP) ITINERARE-Innovative Therapies in Rare Diseases. L.P.G is supported by European Union's Horizon 2020 research and innovation programme under the Marie Skłodowska-Curie grant agreement No. 860977. The funders had no role in study design, data collection, and interpretation, or the decision to submit the work for publication.

Availability of data and material Not applicable.

\section{Declarations}

Conflict of interest The authors declare no competing interests. V.S. is an employee of Nestlé Research, which is part of the Société des Produits Nestlé S.A. (SPN).

Ethics approval and consent to participate Not applicable.

Consent for publication Not applicable.

Open Access This article is licensed under a Creative Commons Attribution 4.0 International License, which permits use, sharing, adaptation, distribution and reproduction in any medium or format, as long as you give appropriate credit to the original author(s) and the source, provide a link to the Creative Commons licence, and indicate if changes were made. The images or other third party material in this article are included in the article's Creative Commons licence, unless indicated otherwise in a credit line to the material. If material is not included in the article's Creative Commons licence and your intended use is not permitted by statutory regulation or exceeds the permitted use, you will need to obtain permission directly from the copyright holder. To view a copy of this licence, visit http://creativecommons.org/licenses/by/4.0/.

\section{References}

1. Altmann R (1890) Die Elementarorganismen und ihre Beziehungen zu den Zellen, 1st edn. Veit, Leipzig

2. Benda C (1898) Ueber die spermatogenese der vertebraten und höherer evertebraten, II. Theil: Die histiogenese der spermien. J Arch Anat Physiol 73:393-398

3. Suomalainen A, Battersby BJ (2018) Mitochondrial diseases: the contribution of organelle stress responses to pathology. Nat Rev Mol Cell Biol 19(2):77-92

4. Giorgi C, Marchi S, Pinton P (2018) The machineries, regulation and cellular functions of mitochondrial calcium. Nat Rev Mol Cell Biol 19(11):713-730

5. Berridge MJ, Lipp P, Bootman MD (2000) The versatility and universality of calcium signalling. Nat Rev Mol Cell Biol 1(1):11-21

6. Giorgi $\mathrm{C}$ et al (2018) Calcium dynamics as a machine for decoding signals. Trends Cell Biol 28(4):258-273

7. Raimundo N (2014) Mitochondrial pathology: stress signals from the energy factory. Trends Mol Med 20(5):282-292

8. Lin MT, Beal MF (2006) Mitochondrial dysfunction and oxidative stress in neurodegenerative diseases. Nature 443(7113):787-795

9. Galluzzi L, Yamazaki T, Kroemer G (2018) Linking cellular stress responses to systemic homeostasis. Nat Rev Mol Cell Biol 19(11):731-745

10. Archer SL (2013) Mitochondrial dynamics-mitochondrial fission and fusion in human diseases. $\mathrm{N}$ Engl $\mathrm{J}$ Med 369(23):2236-2251

11. Sorrentino V, Menzies KJ, Auwerx J (2018) Repairing mitochondrial dysfunction in disease. Annu Rev Pharmacol Toxicol 58:353-389

12. Nunnari J, Suomalainen A (2012) Mitochondria: in sickness and in health. Cell 148(6):1145-1159

13. Schlosser P et al (2020) Genetic studies of urinary metabolites illuminate mechanisms of detoxification and excretion in humans. Nat Genet 52(2):167-176

14. Cheng Y et al (2021) Rare genetic variants affecting urine metabolite levels link population variation to inborn errors of metabolism. Nat Commun 12(1):964

15. Collins TJ et al (2002) Mitochondria are morphologically and functionally heterogeneous within cells. Embo J 21(7):1616-1627

16. Pagliarini DJ et al (2008) A mitochondrial protein compendium elucidates complex I disease biology. Cell 134(1):112-123

17. Morgenstern M et al (2017) Definition of a high-confidence mitochondrial proteome at quantitative scale. Cell Rep 19(13):2836-2852

18. Kauppila TES, Kauppila JHK, Larsson NG (2017) Mammalian mitochondria and aging: an update. Cell Metab 25(1):57-71

19. Lightowlers RN, Taylor RW, Turnbull DM (2015) Mutations causing mitochondrial disease: what is new and what challenges remain? Science 349(6255):1494-1499

20. Scorrano L et al (2019) Coming together to define membrane contact sites. Nat Commun 10(1):1287

21. Giacomello $\mathrm{M}$ et al (2020) The cell biology of mitochondrial membrane dynamics. Nat Rev Mol Cell Biol 21(4):204-224

22. Vogel F et al (2006) Dynamic subcompartmentalization of the mitochondrial inner membrane. J Cell Biol 175(2):237-247 
23. Bhargava P, Schnellmann RG (2017) Mitochondrial energetics in the kidney. Nat Rev Nephrol 13(10):629-646

24. van der Bliek AM, Sedensky MM, Morgan PG (2017) Cell biology of the mitochondrion. Genetics 207(3):843

25. Kunji ERS et al (2020) The slc25 carrier family: important transport proteins in mitochondrial physiology and pathology. Physiology (Bethesda) 35(5):302-327

26. Pfanner N, Warscheid B, Wiedemann N (2019) Mitochondrial proteins: from biogenesis to functional networks. Nat Rev Mol Cell Biol 20(5):267-284

27. Shpilka T, Haynes CM (2018) The mitochondrial UPR: mechanisms, physiological functions and implications in ageing. Nat Rev Mol Cell Biol 19(2):109-120

28. Lill R (2009) Function and biogenesis of iron-sulphur proteins. Nature 460(7257):831-838

29. Wiedemann N, Pfanner N (2017) Mitochondrial machineries for protein import and assembly. Annu Rev Biochem 86(1):685-714

30. Cai X, Xu H, Chen ZJ (2017) Prion-like polymerization in immunity and inflammation. Cold Spring Harb Perspect Biol. https:// doi.org/10.1101/cshperspect.a023580

31. Wang C, Youle RJ (2009) The role of mitochondria in apoptosis*. Annu Rev Genet 43:95-118

32. Fuchs Y, Steller H (2015) Live to die another way: modes of programmed cell death and the signals emanating from dying cells. Nat Rev Mol Cell Biol 16(6):329-344

33. Palikaras K, Lionaki E, Tavernarakis N (2018) Mechanisms of mitophagy in cellular homeostasis, physiology and pathology. Nat Cell Biol 20(9):1013-1022

34. D'Amico D, Sorrentino V, Auwerx J (2017) Cytosolic proteostasis networks of the mitochondrial stress response. Trends Biochem Sci 42(9):712-725

35. Melber A, Haynes CM (2018) UPR(mt) regulation and output: a stress response mediated by mitochondrial-nuclear communication. Cell Res 28(3):281-295

36. Nargund AM et al (2012) Mitochondrial import efficiency of ATFS-1 regulates mitochondrial UPR activation. Science 337(6094):587-590

37. Tremblay BP, Haynes CM (2020) Mitochondrial distress call moves to the cytosol to trigger a response to stress. Nature 579(7799):348-349

38. Durieux J, Wolff S, Dillin A (2011) The cell-non-autonomous nature of electron transport chain-mediated longevity. Cell 144(1):79-91

39. Houtkooper RH et al (2013) Mitonuclear protein imbalance as a conserved longevity mechanism. Nature 497(7450):451-457

40. Zhao Q et al (2002) A mitochondrial specific stress response in mammalian cells. EMBO J 21(17):4411-4419

41. Liu Y et al (2014) Caenorhabditis elegans pathways that surveil and defend mitochondria. Nature 508(7496):406-410

42. Guo $X$ et al (2020) Mitochondrial stress is relayed to the cytosol by an OMA1-DELE1-HRI pathway. Nature 579(7799):427-432

43. Fessler $\mathrm{E}$ et al (2020) A pathway coordinated by DELE1 relays mitochondrial stress to the cytosol. Nature 579(7799):433-437

44. Sorrentino V et al (2017) Enhancing mitochondrial proteostasis reduces amyloid- $\beta$ proteotoxicity. Nature 552(7684):187-193

45. Romani M et al (2021) NAD(+) boosting reduces age-associated amyloidosis and restores mitochondrial homeostasis in muscle. Cell Rep 34(3): 108660

46. Rojas-Morales P et al (2020) Protection against renal ischemia and reperfusion injury by short-term time-restricted feeding involves the mitochondrial unfolded protein response. Free Radic Biol Med 154:75-83

47. Zhu PJ et al (2019) Activation of the ISR mediates the behavioral and neurophysiological abnormalities in Down syndrome. Elife 366(6467):843-849
48. Krukowski K et al (2020) Small molecule cognitive enhancer reverses age-related memory decline in mice. Elife 9:e62048

49. Hato $\mathrm{T}$ et al (2019) Bacterial sepsis triggers an antiviral response that causes translation shutdown. J Clin Investig 129(1):296-309

50. Frezza C et al (2006) OPA1 controls apoptotic cristae remodeling independently from mitochondrial fusion. Cell 126(1):177-189

51. Ballabio A, Bonifacino JS (2020) Lysosomes as dynamic regulators of cell and organismal homeostasis. Nat Rev Mol Cell Biol 21(2):101-118

52. Pickles S, Vigié P, Youle RJ (2018) Mitophagy and quality control mechanisms in mitochondrial maintenance. Curr Biol 28(4):R170-R185

53. Sugiura A et al (2014) A new pathway for mitochondrial quality control: mitochondrial-derived vesicles. Embo J 33(19):2142-2156

54. Levine B, Kroemer G (2019) Biological functions of autophagy genes: a disease perspective. Cell 176(1-2):11-42

55. Pohl C, Dikic I (2019) Cellular quality control by the ubiquitinproteasome system and autophagy. Science 366(6467):818-822

56. Eisner V, Picard M, Hajnóczky G (2018) Mitochondrial dynamics in adaptive and maladaptive cellular stress responses. Nat Cell Biol 20(7):755-765

57. Mizushima N, Levine B (2020) Autophagy in human diseases. N Engl J Med 383(16):1564-1576

58. Jin SM et al (2010) Mitochondrial membrane potential regulates PINK1 import and proteolytic destabilization by PARL. J Cell Biol 191(5):933-942

59. Greene AW et al (2012) Mitochondrial processing peptidase regulates PINK1 processing, import and Parkin recruitment. EMBO Rep 13(4):378-385

60. Harper JW, Ordureau A, Heo JM (2018) Building and decoding ubiquitin chains for mitophagy. Nat Rev Mol Cell Biol 19(2):93-108

61. Lazarou $\mathrm{M}$ et al (2015) The ubiquitin kinase PINK1 recruits autophagy receptors to induce mitophagy. Nature 524(7565):309-314

62. Shin J-H et al (2011) PARIS (ZNF746) Repression of PGC-1 $\alpha$ contributes to neurodegeneration in Parkinson's disease. Cell 144(5):689-702

63. Lee Y et al (2017) PINK1 primes Parkin-mediated ubiquitination of PARIS in dopaminergic neuronal survival. Cell Rep 18(4):918-932

64. Fang EF et al (2014) Defective mitophagy in XPA via PARP-1 hyperactivation and NAD+/SIRT1 reduction. Cell 157(4):882-896

65. Liang D et al (2020) SIRT1/PGC-1 pathway activation triggers autophagy/mitophagy and attenuates oxidative damage in intestinal epithelial cells. Biochimie 170:10-20

66. Liu L et al (2021) Mitophagy receptor FUNDC1 is regulated by PGC- $1 \alpha /$ NRF1 to fine tune mitochondrial homeostasis. EMBO Rep 22(3):50629

67. Vainshtein A et al (2015) Role of PGC-1 $\alpha$ during acute exercise-induced autophagy and mitophagy in skeletal muscle. Am J Physiol Cell Physiol 308(9):C710-C719

68. Rikka S et al (2011) Bnip3 impairs mitochondrial bioenergetics and stimulates mitochondrial turnover. Cell Death Differ 18(4):721-731

69. Novak I et al (2010) Nix is a selective autophagy receptor for mitochondrial clearance. EMBO Rep 11(1):45-51

70. Liu L et al (2012) Mitochondrial outer-membrane protein FUNDC1 mediates hypoxia-induced mitophagy in mammalian cells. Nat Cell Biol 14(2):177-185

71. Sandoval $\mathrm{H}$ et al (2008) Essential role for Nix in autophagic maturation of erythroid cells. Nature 454(7201):232-235 
72. Chu CT et al (2013) Cardiolipin externalization to the outer mitochondrial membrane acts as an elimination signal for mitophagy in neuronal cells. Nat Cell Biol 15(10):1197-1205

73. Rahman J, Rahman S (2018) Mitochondrial medicine in the omics era. Lancet 391(10139):2560-2574

74. Rahman S (2020) Mitochondrial disease in children. J Intern Med 287(6):609-633

75. Gorman GS et al (2015) Prevalence of nuclear and mitochondrial DNA mutations related to adult mitochondrial disease. Ann Neurol 77(5):753-759

76. Pitceathly RDS et al (2021) Moving towards clinical trials for mitochondrial diseases. J Inherit Metab Dis 44(1):22-41

77. Vella F (1996) The metabolic and molecular bases of inherited disease, 7th edn. In: Sriver CR, Beaudet AL, Sly WS, Valle D (eds) P 4605. McGraw-Hill, New York. ISBN 0-07-909826-6. Biochemical Education, vol 24, issue 1, pp 65-65

78. Dimitrov B et al (2021) Organic acidurias: major gaps, new challenges, and a yet unfulfilled promise. J Inherit Metab Dis 44(1):9-21

79. Haijes HA et al (2019) Pathophysiology of propionic and methylmalonic acidemias. Part 1: complications. J Inherit Metab Dis 42(5):730-744

80. Sniderman LC et al (1999) Outcome of individuals with lowmoderate methylmalonic aciduria detected through a neonatal screening program. J Pediatr 134(6):675-680

81. Forny P et al (2016) Molecular genetic characterization of 151 Mut-Type methylmalonic aciduria patients and identification of 41 novel mutations in MUT. Hum Mutat 37(8):745-754

82. Worgan LC et al (2006) Spectrum of mutations in mut methylmalonic acidemia and identification of a common Hispanic mutation and haplotype. Hum Mutat 27(1):31-43

83. Mellon AF et al (2000) Effect of oral antibiotics on intestinal production of propionic acid. Arch Dis Child 82(2):169-172

84. Forny P et al (2021) Guidelines for the diagnosis and management of methylmalonic acidaemia and propionic acidaemia: First revision. J Inherit Metab Dis. https://doi.org/10.1002/ jimd. 12370

85. Dobson CM et al (2002) Identification of the gene responsible for the cblA complementation group of vitamin B12-responsive methylmalonic acidemia based on analysis of prokaryotic gene arrangements. Proc Natl Acad Sci USA 99(24):15554-15559

86. Dobson CM et al (2002) Identification of the gene responsible for the cblB complementation group of vitamin B12-dependent methylmalonic aciduria. Hum Mol Genet 11(26):3361-3369

87. Coelho D et al (2008) Gene identification for the cblD defect of vitamin B12 metabolism. N Engl J Med 358(14):1454-1464

88. Dobson CM et al (2006) Homozygous nonsense mutation in the MCEE gene and siRNA suppression of methylmalonyl-CoA epimerase expression: a novel cause of mild methylmalonic aciduria. Mol Genet Metab 88(4):327-333

89. Waters PJ et al (2016) Methylmalonyl-coA epimerase deficiency: A new case, with an acute metabolic presentation and an intronic splicing mutation in the MCEE gene. Mol Genet Metab Rep 9:19-24

90. Carrozzo R et al (2016) Succinate-CoA ligase deficiency due to mutations in SUCLA2 and SUCLG1: phenotype and genotype correlations in 71 patients. J Inherit Metab Dis 39(2):243-252

91. Sloan JL et al (2011) Exome sequencing identifies ACSF3 as a cause of combined malonic and methylmalonic aciduria. Nat Genet 43(9):883-886

92. Levtova A et al (2019) Combined malonic and methylmalonic aciduria due to ACSF3 mutations: Benign clinical course in an unselected cohort. J Inherit Metab Dis 42(1):107-116

93. de Keyzer $\mathrm{Y}$ et al (2009) Multiple OXPHOS deficiency in the liver, kidney, heart, and skeletal muscle of patients with methylmalonic aciduria and propionic aciduria. Pediatr Res 66(1):91-95
94. Saudubray JM, Cazorla AG (2016) Clinical approach to inborn errors of metabolism in pediatrics. In: Saudubray JM, Baumgartner MR, Walter J (eds) Inborn metabolic diseases. Springer, Berlin, pp 3-69

95. Fenton W, Gravel R, Rosenblatt D (2001) Disorders of propionate and methylmalonate metabolism. In: Scriver CR et al (eds) The metabolic and molecular bases of inherited disease. McGraw-Hill Book Co, New York, pp 2165-2193

96. Horster $\mathrm{F}$ et al (2007) Long-term outcome in methylmalonic acidurias is influenced by the underlying defect (mut0, mut-, cblA, cblB). Pediatr Res 62(2):225-230

97. Baumgartner MR et al (2014) Proposed guidelines for the diagnosis and management of methylmalonic and propionic acidemia. Orphanet J Rare Dis 9:130

98. Zwickler T et al (2008) Diagnostic work-up and management of patients with isolated methylmalonic acidurias in European metabolic centres. J Inherit Metab Dis 31(3):361-367

99. Ktena YP et al (2015) Delineating the spectrum of impairments, disabilities, and rehabilitation needs in methylmalonic acidemia (MMA). Am J Med Genet A 167(9):2075-2084

100. Nizon $M$ et al (2013) Long-term neurological outcome of a cohort of 80 patients with classical organic acidurias. Orphanet J Rare Dis 8:148

101. Kovacevic A et al (2020) Cardiac phenotype in propionic acidemia - results of an observational monocentric study. Mol Genet Metab 130(1):41-48

102. Radmanesh A et al (2008) Methylmalonic acidemia: brain imaging findings in 52 children and a review of the literature. Pediatr Radiol 38(10): 1054

103. Kanaumi $T$ et al (2006) Neuropathology of methylmalonic acidemia in a child. Pediatr Neurol 34(2):156-159

104. Yang L et al (2020) Brain MRI features of methylmalonic acidemia in children: the relationship between neuropsychological scores and MRI findings. Sci Rep 10(1):13099

105. Harting I et al (2008) Looking beyond the basal ganglia: The spectrum of MRI changes in methylmalonic acidaemia. J Inherit Metab Dis 31(3):368

106. Brismar J, Ozand PT (1994) CT and MR of the brain in disorders of the propionate and methylmalonate metabolism. Am J Neuroradiol 15(8):1459-1473

107. Kolker $\mathrm{S}$ et al (2015) The phenotypic spectrum of organic acidurias and urea cycle disorders. Part 2: the evolving clinical phenotype. J Inherit Metab Dis 38(6):1059-1074

108. Mink JW (2003) The basal ganglia and involuntary movements: impaired inhibition of competing motor patterns. Arch Neurol 60(10):1365-1368

109. Heidenreich R et al (1988) Acute extrapyramidal syndrome in methylmalonic acidemia: "Metabolic stroke" involving the globus pallidus. J Pediatr 113(6):1022-1027

110. de Sousa C et al (1989) Focal changes in the globi pallidi associated with neurological dysfunction in methylmalonic acidaemia. Neuropediatrics 20(4):199-201

111. Korf B, Wallman JK, Levy HL (1986) Bilateral lucency of the globus pallidus complicating methylmalonic acidemia. Ann Neurol 20(3):364-366

112. Di Giovanna AP et al (2018) Whole-brain vasculature reconstruction at the single capillary level. Sci Rep 8(1):12573

113. Shih Y-YI et al (2011) Striatal and cortical BOLD blood flow, blood volume, oxygen consumption, and glucose consumption changes in noxious forepaw electrical stimulation. J Cereb Blood Flow Metab 31(3):832-841

114. Kirst $\mathrm{C}$ et al (2020) Mapping the fine-scale organization and plasticity of the brain vasculature. Cell 180(4):780-795.e25

115. Misgeld T, Schwarz TL (2017) Mitostasis in neurons: maintaining mitochondria in an extended cellular architecture. Neuron 96(3):651-666 
116. Shepherd GMG, Harris KM (1998) Three-dimensional structure and composition of $\mathrm{CA} 3 \rightarrow \mathrm{CA} 1$ axons in rat hippocampal slices: implications for presynaptic connectivity and compartmentalization. J Neurosci 18(20):8300-8310

117. Bolam JP, Pissadaki EK (2012) Living on the edge with too many mouths to feed: Why dopamine neurons die. Mov Disord 27(12):1478-1483

118. Hegde AN et al (2011) Differential diagnosis for bilateral abnormalities of the basal ganglia and thalamus. Radiographics 31(1):5-30

119. Kennedy $\mathrm{C}$ et al (1978) Local cerebral glucose utilization in the normal conscious macaque monkey. Nature 4(4):293-301

120. Attwell D, Laughlin SB (2001) An energy budget for signaling in the grey matter of the brain. J Cereb Blood Flow Metab 21(10):1133-1145

121. Baker EH et al (2015) MRI Characteristics of globus pallidus infarcts in isolated methylmalonic acidemia. Am J Neuroradiol 36(1):194

122. Martinez Alvarez L et al (2016) Optic neuropathy in methylmalonic acidemia and propionic acidemia. Br J Ophthalmol 100(1):98-104

123. Cosson MA et al (2009) Long-term outcome in methylmalonic aciduria: a series of 30 French patients. Mol Genet Metab 97(3):172-178

124. Hörster F et al (2009) Prediction of outcome in isolated methylmalonic acidurias: combined use of clinical and biochemical parameters. J Inherit Metab Dis 32(5):630

125. Chandler RJ et al (2009) Mitochondrial dysfunction in mut methylmalonic acidemia. FASEB J 23(4):1252-1261

126. Manoli I et al (2013) Targeting proximal tubule mitochondrial dysfunction attenuates the renal disease of methylmalonic acidemia. Proc Natl Acad Sci USA 110(33):13552-13557

127. Kruszka PS et al (2013) Renal growth in isolated methylmalonic acidemia. Genet Med 15(12):990-996

128. Ruppert $\mathrm{T}$ et al (2015) Molecular and biochemical alterations in tubular epithelial cells of patients with isolated methylmalonic aciduria. Hum Mol Genet 24(24):7049-7059

129. Manoli I et al (2018) FGF21 underlies a hormetic response to metabolic stress in methylmalonic acidemia. JCI Insight. https:// doi.org/10.1172/jci.insight.124351

130. Luciani A et al (2020) Impaired mitophagy links mitochondrial disease to epithelial stress in methylmalonyl-CoA mutase deficiency. Nat Commun 11(1):970

131. Luciani A, Devuyst O (2020) Methylmalonyl acidemia: from mitochondrial metabolism to defective mitophagy and disease. Autophagy 16(6):1159-1161

132. Chen Z, Berquez M, Luciani A (2020) Mitochondria, mitophagy, and metabolic disease: towards assembling the puzzle. Cell Stress 4(6): 147-150

133. Luciani A, Freedman BS (2020) Induced pluripotent stem cells provide mega insights into kidney disease. Kidney Int 98(1):54-57

134. Baumgartner MR, Froese DS (2020) Lysosomal Vitamin B12 trafficking. In: Gasnier B, Zhu MX (eds) Ion and molecule transport in lysosomes. Taylor \& Francis Group, Boca Raton, pp 161-176

135. Anzmann AF et al (2019) Multi-omics studies in cellular models of methylmalonic acidemia and propionic acidemia reveal dysregulation of serine metabolism. Biochim Biophys Acta Mol Basis Dis 1865(12): 165538

136. Richard E et al (2018) Generation and characterization of two human iPSC lines from patients with methylmalonic acidemia cblB type. Stem Cell Res 29:143-147

137. Forny $\mathrm{P}$ et al (2019) Liver neoplasms in methylmalonic aciduria: An emerging complication. J Inherit Metab Dis 42(5):793-802
138. Collado MS et al (2020) Biochemical and anaplerotic applications of in vitro models of propionic acidemia and methylmalonic acidemia using patient-derived primary hepatocytes. Mol Genet Metab 130(3):183-196

139. Mishra J et al (2003) Identification of neutrophil gelatinase-associated lipocalin as a novel early urinary biomarker for ischemic renal injury. J Am Soc Nephrol 14(10):2534-2543

140. Wang $Y$ et al (2007) Lipocalin-2 is an inflammatory marker closely associated with obesity, insulin resistance, and hyperglycemia in humans. Clin Chem 53(1):34-41

141. Fakruddin $M$ et al (2018) Defective mitochondrial tRNA taurine modification activates global proteostress and leads to mitochondrial disease. Cell Rep 22(2):482-496

142. Peters $\mathrm{H}$ et al (2003) A knock-out mouse model for methylmalonic aciduria resulting in neonatal lethality. J Biol Chem 278(52):52909-52913

143. Chandler RJ et al (2007) Metabolic phenotype of methylmalonic acidemia in mice and humans: the role of skeletal muscle. BMC Med Genet 8:64

144. Murphy GE et al (2010) Ion-abrasion scanning electron microscopy reveals distorted liver mitochondrial morphology in murine methylmalonic acidemia. J Struct Biol 171(2):125-132

145. Peters HL et al (2012) Mouse models for methylmalonic aciduria. PLoS ONE 7(7):e40609

146. Buck NE et al (2012) Development of transgenic mice containing an introduced stop codon on the human methylmalonyl-CoA mutase locus. PLoS ONE 7(9):e44974

147. Pavuluri K et al (2019) Noninvasive monitoring of chronic kidney disease using $\mathrm{pH}$ and perfusion imaging. Sci Adv 5(8):eaaw8357

148. Forny P et al (2016) Novel mouse models of methylmalonic aciduria recapitulate phenotypic traits with a genetic dosage effect. J Biol Chem 291(39):20563-20573

149. Wongkittichote $\mathrm{P}$ et al (2019) Tricarboxylic acid cycle enzyme activities in a mouse model of methylmalonic aciduria. Mol Genet Metab 128(4):444-451

150. Lucienne $\mathrm{M}$ et al (2020) In-depth phenotyping reveals common and novel disease symptoms in a hemizygous knock-in mouse model (Mut-ko/ki) of mut-type methylmalonic aciduria. Biochim Biophys Acta Mol Basis Dis 1866(3):165622

151. Lucienne $\mathrm{M}$ et al (2021) Decrease of disease-related metabolites upon fasting in a hemizygous knock-in mouse model (Mut-ko/ki) of methylmalonic aciduria. JIMD Rep 58(1):44-51

152. Madeo F et al (2018) Spermidine in health and disease. Science 359(6374): eaan2788

153. Madeo F et al (2019) Caloric restriction mimetics against ageassociated disease: targets, mechanisms, and therapeutic potential. Cell Metab 29(3):592-610

154. Fang EF et al (2019) Mitophagy inhibits amyloid- $\beta$ and tau pathology and reverses cognitive deficits in models of Alzheimer's disease. Nat Neurosci 22(3):401-412

155. Armstrong AJ et al (2021) A novel small molecule approach for the treatment of propionic and methylmalonic acidemias. Mol Genet Metab 133:71-82

156. Armstrong AJ et al (2021) Identification of 2,2-dimethylbutanoic acid (HST5040), a clinical development candidate for the treatment of propionic acidemia and methylmalonic acidemia. J Med Chem 64:5037-5048

157. Chandler RJ, Venditti CP (2008) Adenovirus-mediated gene delivery rescues a neonatal lethal murine model of mut( 0$)$ methylmalonic acidemia. Hum Gene Ther 19(1):53-60

158. Chandler RJ, Venditti CP (2010) Long-term rescue of a lethal murine model of methylmalonic acidemia using adeno-associated viral gene therapy. Mol Ther 18(1):11-16

159. Chandler RJ, Venditti CP (2012) Pre-clinical efficacy and dosing of an AAV8 vector expressing human methylmalonyl-CoA 
mutase in a murine model of methylmalonic acidemia (MMA). Mol Genet Metab 107(3):617-619

160. Carrillo-Carrasco N et al (2010) Liver-directed recombinant adeno-associated viral gene delivery rescues a lethal mouse model of methylmalonic acidemia and provides long-term phenotypic correction. Hum Gene Ther 21(9):1147-1154

161. Senac JS et al (2012) Gene therapy in a murine model of methylmalonic acidemia using rAAV9-mediated gene delivery. Gene Ther 19(4):385-391

162. Wong ES et al (2014) Correction of methylmalonic aciduria in vivo using a codon-optimized lentiviral vector. Hum Gene Ther 25(6):529-538

163. Chandler RJ et al (2021) Promoterless, nuclease-free genome editing confers a growth advantage for corrected hepatocytes in mice with methylmalonic acidemia. Hepatology 73(6):2223-2237

164. An D et al (2018) Systemic messenger RNA therapy as a treatment for methylmalonic acidemia. Cell Rep 24(9):2520

165. An D et al (2019) Long-term efficacy and safety of mRNA therapy in two murine models of methylmalonic acidemia. EBioMedicine 45:519-528
166. Billia F et al (2011) PTEN-inducible kinase 1 (PINK1)/Park6 is indispensable for normal heart function. Proc Natl Acad Sci 108(23): 9572

167. Gong $\mathrm{Y}$ et al (2015) High-speed recording of neural spikes in awake mice and flies with a fluorescent voltage sensor. Science 350(6266): 1361-1366

168. Oh C-K et al (2017) S-Nitrosylation of PINK1 atenuates PINK1/ Parkin-dependent mitophagy in hiPSC-based Parkinson's disease models. Cell Rep 21(8):2171-2182

169. Gomes AP et al (2020) Age-induced accumulation of methylmalonic acidemia promotes tumor progression. Nature 585:283-287

Publisher's Note Springer Nature remains neutral with regard to jurisdictional claims in published maps and institutional affiliations. 